\title{
Principios bioéticos en salud pública: limitaciones y propuestas
}

\author{
Bioethical principles in public health: \\ limitations and proposals
}

Fermin Roland Schramm 1

Miguel Kottow 2,3

\footnotetext{
1 Departamento de Ciências Sociais, Escola Nacional de Saú de Pública, Fun dação Oswaldo Cruz. Rua Leopol do Bulhoes 1480, sal a 914, Ri o de Janeiro, RJ 21041-210, Brasi roland@ensp.fiocruz.br rolandschram@yahoo.com.br 2 Facultad de Medicina, Universidad de Chile. Av. Independencia 1027, Santiago, Chile. 3 Departamento deFilosofía, Facultad de Filosofía y Humanidades, Universidad de Chile. Casilla 16168, Correo 9, Santiago, Chile. guarvie@ctcreuma.c
}

Abstract We propose to analyze the specificity of ethical problems in public health issues and to el ucidate the applicability of principlism as a problem-solving strategy in this realm. Although well-established in clinical ethics, principlism is not an adequate model to be used in public health, sinceit is basically intended to serveas a moral guide in the physician-patient encounter. We discuss the possi ble adequacy of principles like "solidarity", "ontic responsibility" (as proposed by Jonas), and "caring or diaconal responsibility" as presented by Lévinas. Solidarity appears to be insufficiently specified, whereas the other two perspectives may be adapted to public heal th issues by bringing together Jonas' ontological and Lévinas' transcendental concerns to form a principle of protection that might better serve the purposes of such an ethics. This principle would hel p to identify more clearly the goals and agents involved in the implementation of public policies that are expected to beboth morally correct and pragmatically effective.

Key words Bioethics; Morals; Public Policy

Resumen Esteartículo intenta caracterizar la especificidad delos problemas morales en salud pública y analizar la aplicabilidad del modelo principialista como padrón para dirimir sus conflictos. Aunque considerado perti nente para la bi oética clínica, este modelo no es aplicablesin más a los dilemas en salud pública, puesto que se fundamenta en la moral delas interrelaciones médico-paciente. Se anal iza la pertinencia de los princi pi os de "solidaridad", de "responsabilidad óntica" según Jonas, y de "responsabilidad diacónica" según Lévinas, destacando la i naplicabilidad del primero y la posible adaptación delos otros dos a la salud pública. A ese respecto se discute la posibilidad de vincular la preocupación ontológi ca de Jonas y la trascendental de Lévinas, proponi endo un principio de protección que sería más adecuado a los propósitos de una ética dela salud pública, permitiendo identificar claramentelos objeti vos y los actores implicados en una implementación de políticas públicas moralmentecorrectasy pragmáticamente efectivas.

Palabras clave Bioética; Principios Morales; Política Social 


\section{Introducción}

La especificidad de los problemas morales que se presentan en salud pública plantea la siguiente pregunta: zacaso la bioética ha desarrollado las herramientas adecuadas para enfrentar los principales dilemas morales que se dan en programas y prácticas de salud colectiva? Allí donde estos dilemas han sido abordados, la mayoría de los autores suelen adaptar los instrumentos conceptuales de la bioética clínica, en analogía a los modelos aplicados a la relación médico/paciente, estrategia que ha sido criticada en diversas publicaciones (Lachmann, 1998; Skrabanek, 1990).

Se tiende a adaptar al contexto colectivo el modelo de los cuatro principios de "no-maleficencia", "beneficencia", "autonomía" y "justicia" (Beauchamp \& Childress, 1994).

Este modelo ha sido ampliamente discutido y criticado (Clouser \& Gert, 1990; Evans, 2000; Gillon, 1996; Jonsen \& Toulmin, 1988; Kuczewski, 1998), y esto, sin embargo, no nos permite avanzar en el tema que aquí nos preocupa. De hecho, todas las disquisiciones han ignorado el ámbito de la moral en salud pública y sus aspectos particulares, aun cuando se ha considerado algún problema específico como la ética de la prevención de infección por $\mathrm{VIH}$, algún programa de detección y tratamiento de predisposiciones, o el "derecho a salud". Ello ha ocurrido en forma genérica, sin atender a los fundamentos y métodos capaces de analizar y validar los dilemas y las propuestas específicas al ámbito de la salud pública.

El presente artículo explora una posible ética en salud pública que sea más atinente a los conflictos morales que surgen en este campo. En la primera parte se presentan las inadecuaciones del principialismo para el ámbito de la salud pública. La segunda parte aborda tres propuestas alternativas y aparentemente más adecuadas: la propuesta de una ética de solidaridad revitalizada por Callahan (1998), así como dos versiones de ética de la responsabilidad: la ética de la responsabilidad óntica de Jonas (1979) y la ética de responsabilidad diacónica de Lévinas (1974). Después de mostrar las insuficiencias de la primera, se propone utilizar elementos comunes de ambas formas de ética de responsabilidad presentadas, sugi riendo una nueva versión de ética de responsabilidad elaborada como un principio de protección, que no había sido contemplado como tal por el pensamiento bioético tradicional.

El principio de protección será aquí propuesto como el más adecuado para enfrentar los problemas moral es relacionados con la sa- lud pública, capaz de generar acuerdos entre salubristas y eticistas cuando enfrentan conflictos que no pueden dirimirse con recurso al modelo principialista.

\section{Inadecuación del modelo principialista}

Clásicamente se ha planteado la coexistencia de cuatro principios: beneficencia, no-maleficencia, autonomía y justicia, enunciados para la investigación en seres humanos (The National Commission for the Protection of Human Subjects of Biomedical and Behavioral Research, 1978) y bioética clínica (Beauchamp \& Childress, 1994). La beneficencia presupone que todo acto médico tiene por finalidad hacer el bien y cuidar los intereses del paciente; la no-maleficencia señala que toda intervención debe evitar o reducir al mínimo los riesgos y daños para el afectado; la autonomía requiere que todos los participantes en el acto biomédico consientan informada y voluntariamente al proyecto terapéutico o de investigación a realizarse; según la justicia, todos los recursos, derechos y obligaciones deben ser ecuánimemente distribuidos y respetados para cada uno.

Esta breve descripción deja en claro que estos lineamientos éticos se refieren a interacciones entre individuos, tal como se da ejemplarmente en la relación médico-paciente o en la de investigador-probando.

La incorporación de avances técnicos y científicos al campo de las ciencias de la vida y de la salud, la transición epidemiológica que afecta a todas las sociedades tanto "desarrolladas" como "en desarrollo", y asimismo la emergencia de una demanda calificada de servicios de salud, van paulatinamente acarreando nuevas cuestiones éticas también para el campo de la salud pública. Ello lleva a indagar acaso, más allá de una postura general de "un mínimo digno de cuidados de sal ud" supuestamente garantizado por el Estado y sus instituciones políticas y jurídicas (Beauchamp \& Childress, 1994; Daniels, 1985), existe de hecho la posibilidad concreta, económica y socialmente sustentable de extender la prevención y la protección contra enfermedades, así como la promoción de estilos de vida más saludables para toda la población, garantizando que una opinión pública informada y en ejercicio de sus derechos ciudadanos eval úe y controle estas instituciones y garantice los derechos comprometidos.

Salud pública y biomedicina clínica tienen cada una su propia especificidad teórica, práctica y normativa, a pesar de compartir la misma categoría genérica "salud" y tener en común una serie de preocupaciones relativas al bienes- 
tar humano desde el punto de vista de las enfermedades, su tratamiento y su eventual curación. A diferencia de la biomedicina clínica, el referente universal de la salud pública son las medidas colectivas de prevención que no necesariamente pasan por la relación interpersonal entre médico y paciente o, si se prefiere, entre prestador de servicios y usuario. Se refieren estas medidas a poblaciones humanas y a sus contextos socio-ambientales por un lado, y a instituciones públicas responsables por la implementación de políticas de prevención y promoción de salud por el otro. El especialista en salud pública podrá estar de acuerdo que existen prima facie interacciones y aun sinergias entre el campo de la biomedicina clínica y la salud pública, rechazando por lo tanto el separarlas en "dos culturas" que se ignoran mutuamente (Coye, 1994). También en este contexto parecen insuficientes las herramientas del modelo principialista para enfrentar los dilemas que actualmente ocupan la salud pública.

La reducción de la moralidad en salud pública al ámbito de la ética biomédica clínica es criticable porque:

- Descuida la especificidad de los problemas que enfrenta la salud pública, preocupada tanto de la prevención de morbilidades en poblaciones humanas como de la promoción en salud y de la calidad del ambiente biológico, psicológico, social y cultural;

- Reducela complejidad de las relaciones entre biomedicina clínica y salud pública, lo cual implica que no todo lo relevante en el ámbito bioético clínico lo es también desde el punto de vista de la ética en salud pública.

En políticas públicas se trabaja con riesgos y complicaciones de grandes magnitudes así como difícilmente cuantificables, razón por la cual se vuelve extremadamente difícil hacer predicciones que posibiliten orientar moralmente a través del modelo principialista los comportamientos apropiados para minimizar Ios efectos negativos; por ende tal modelo debe ser considerado inadecuado para enfrentar los dilemas morales en salud pública.

Los problemas éticos en salud pública no se dejan enmarcar adecuadamente en el pensamiento bioético actual inspirado en el modelo principialista y, más allá, la evaluación ética de políticas sanitarias, sobre todo de carácter preventivo, puede llevar a una especie de "tiranía sanitarista" (Becker, 1986) y a un "fanatismo preventivista" (Skrabanek, 1994), por lo cual es preciso dar con una ética específica para salud pública.
Propuestas alternativas para una ética en salud pública

Cabe la pregunta: ¿acaso existen otros principios como el de solidaridad o el de responsabilidad, candidatos de peso como modelo de análisis ético aplicado a salud pública?

\section{El principio de solidaridad}

La solidaridad aparece en el siglo XVIII en el pensamiento sociopolítico de Francia y es propugnado en las encíclicas sociales del período leonino y en las de Juan Pablo II. La solidaridad asume quela atomización de un colectivo hace más vulnerable a cada uno de sus miembros, por lo cual es recomendable entender este colectivo como un sólido (=solidaridad) que aúna los esfuerzos de todos para paliar infortunios, defenderse de agresiones, constituir un sistema de protección y elaborar en conjunto para la obtención de bienes comunes. Implícita en la solidaridad está la igualdad, tanto de esfuerzo realizado como de protección obtenida, y allí donde esta simetría se rompe, aparecen los abusos y las desprotecciones de los más débiles.

Callahan (1998) señala que el "principio de solidaridad" constituye uno de los principales ingredientes para poder pensar una medicina que sea al mismo tiempo equitativa y sustentable. Sin embargo, el principio de solidaridad se expone a in numerables críticas, sobre todo allí donde se le utiliza para legitimar políticas de asignación de recursos, que son reconocidamente finitos y escasos en cualquier sistema sanitario.

El principio de solidaridad aplicado a las políticas públicas es insuficiente para resolver los complejos problemas de salud por estar obligado a ser solidario con los otros en situaciones de profunda diversidad de necesidades y aunque existan discrepancias profundas de valores que quedan opacas y negadas a la argumentación.

\section{Ética de la responsabilidad}

El otro principio a considerar como posible herramienta apropiada para el análisis de cuestiones morales en salud pública es la responsabilidad. Responsabilidad viene del verbo responder y significa en ética hacerse cargo de lo realizado frente al afectado, corolario de lo cual puede ser el cuidar de otro ser humano cuando ese está amenazado en su vulnerabilidad. La 
ética aplicada atribuye responsabilidades a los individuos a partir del presupuesto que cada acto humano ha sido libremente elegido por un agente moral, quien siempre es responsable por sus decisiones y las consecuencias de ellas. Así como la libertad, también la responsabilidad está en la raíz de la ética como elemento constitutivo, siendo exigible sin que sea explícitamente formulada como un principio adicional.

A primera vista parecería que la ética de responsabilidad de Jonas (1979) se puede hacer cargo de los problemas morales en salud pública, visto que el autor pone en la base de su ética el deber de responsabilidad con la totalidad del ser, lo que llamamos de responsabilidad óntica, preservándolo de los procesos de aniquilamiento. Eso significa actuar de tal forma que los actos no sean destructivos para las futuras posibilidades de una vida digna sobre la tierra; el nuevo imperativo moral se refiere por ende más a una política pública que al comportamiento privado. Al menos tres consideraciones tornan impracticable esta propuesta.

No es posible ignorar, en primer término, que la responsabilidad en cuanto principio moral se refiere a entes que "deben ser de carácter personal" (Ferrater-Mora, 1999:3082), no pudiendo por ende guiar actos que carecen de actores identificables, como sería el caso de las políticas de salud pública diseñadas por entidades institucionales y destinadas a una población no siempre claramente acotada. Al respecto cabe preguntar si, al requerir la responsabilidad con un genérico "ser" de la naturaleza y de futuras generaciones, Jonas busca asignar responsabilidades por todo lo que acontece en el mundo. Si así fuese, sería un tipo utópico de responsabilidad, inapropiado y poco convincente para exigir una supererogación moral difícilmente alcanzable. Alternativamente, el principio de responsabilidad podría referirse ante todo a los deberes de las élites gobernantes, conforme a una jerarquía intuitiva que asigna mayor responsabilidad a quien supuestamente tiene más saber y poder. Ello sólo sería razonable si los poderes públicos efectivamente tomaran decisiones desde el punto de vista de la justicia distributiva y de políticas compensatorias. Sin embargo, estas consideraciones se vuelven extremadamente problemáticas y nebulosas al intentar aplicarlas a colectivos y a la sociedad.

En segundo término, Jonas enfoca la responsabilidad en situaciones de incertidumbre (como son la mayoría de las situaciones a que él se refiere: el desarrollo de las biotécnicas, el deterioro ambiental o de las condiciones de vi- da de futuras generaciones), que exigen una posición de prudencia en nuestros actos y de frugalidad en las tentativas de satisfacer nuestras pretensiones, lo que a su vez es inadecuado por defecto. Una política de restricción de inversión en desarrol lo tecnológico, según Jonas, implicaría consecuencias incalculables para la sociedad, comenzando por el desempleo masivo y, desde el punto de vista de la asignación de recursos en salud, una menor cobertura para los más desprotegidos. En consecuencia, este tipo de responsabilidad tampoco puede dar cuenta de acciones de salud colectiva.

En tercer término, es razonable preguntarse cuál sería la confiabilidad de técnicas de diagnóstico y pronósticas desarrolladas en el campo de la biomedicina clínica y aplicadas en el ámbito de la salud colectiva, cada vez que se pretenda detectar vínculos causales entre actos individuales y consecuencias colectivas a fin de asi gnar claramente las responsabilidades involucradas. Al carecer de datos confiables para evaluar acciones sanitarias, la responsabilidad no puede funcion ar como un principio moral legítimo, puesto que no contamos con datos cuantitativos y valóricos fidedignos a los cuales aplicar el análisis moral.

Por supuesto, lo anterior no pretende sugerir que las acciones públicas estén liberadas de las responsabilidades individuales de los actores sociales que actúan en nombre del bien público, bajo pena de clasificarlos como agentes irresponsables. El agente público es también un individuo que, en tanto tal, es agente moral y debe responder por los costos sociales de sus decisiones públicas. Es igualmente cierto, no obstante, que el agente público, por su status profesional otorgado colectivamente, enfrenta serias limitaciones institucionales en sus decisiones y acciones, aun cuando ello no significa que se torne un mero ejecutor de órdenes. De cualquier forma, la cuestión de conservar e identificar una responsabilidad individual en acciones colectivas es un asunto complejo que la bioética aún no ha abordado satisfactoriamente.

Con un poco de imaginación es posible detectar una diversidad de tipos de responsabilidad, desde una responsabilidad por causas y efectos probabilísticamente identificables, hasta responsabilidades imputables al carácter moral del agente. Estos tipos de responsabilidad indican que un agente moral debe ser responsable por las consecuencias de sus actos ante la sociedad y de acuerdo con normas validadas por una instancia evaluadora. En la práctica, no obstante, ello es difícil de realizar debido a las incertidumbres que afectan los 
cálculos probabilísticos utilizados en salud pública, como muestran prácticamente todos los estudios epidemiológicos (Skrabanek, 1992).

Responsabilidad óntica

y responsabilidad diacónica

De los diversos tipos de responsabilidad reconocidos en la literatura, dos parecen ser los candidatos más fuertes para una ética en salud pública: (1) La responsabilidad óntica (o "responsabilidad para con el ser") de Hans Jonas (1979), y (2) la responsabilidad diacónica (o "responsabilidad para con el otro") de Emmanuel Lévinas (1974).

Responsabilidad óntica y responsabilidad diacónica tienen características comunes: no son fruto de una solicitud explícita sino de la vulnerabilidad del sujeto que insinúa una necesidad de amparo que es ofrecido por el agente moral. Ambas son asumidas libremente o, a lo sumo, obedeciendo a un deber imperfecto o virtud relativa a un "perfeccionamiento" de la conducta moral individual, por lo cual no pueden ser consideradas deberes propiamente dichos en sociedades seculares y laicas. Finalmente, los modos de responsabilidad asumidos constituyen un compromiso general que no puede tener objetivos claramente identificados, careciendo de un programa o agenda definidos en cuanto a beneficios específicos propuestos.

¿Qué es lo que decide el agente cuando se ciñe a una de estas formas de responsabilidad? De hecho, nada específico está siendo solicitado y ninguna acción en particular es requerida, no existiendo obligación alguna que esté siendo invocada. A este respeto, Jonas indica que la situación arquetípica que lleva a asumir la responsabilidad óntica es la de los padres frente al recién nacido, y Lévinas cita como ejemplo de responsabilidad diacónica el caso del otro desamparado que me incita a asumir una responsabilidad incondicional... ide protegerlo! No es posible equiparar la responsabilidad presentada por Jonas con la responsabilidad del gestor de políticas públicas, so pena de redundar en una cierta dosis de paternalismo (Goffi, 1997). En el caso de Lévinas, el análisis es más sutil, pues el otro me convoca a asumir por él una responsabilidad incondicional que sólo puede darse en una relación asimétrica y no de reciprocidad; lo cual implica que el yo se define éticamente en función del otro. Bajo esas condiciones de una relación ética individual es difícil entender cómo una responsabilidad diacónica podría transladarse al ámbito de las políticas públicas de prevención y promoción.
Si ninguna acción concreta está indicada para este tipo de oferta libre y de responsabilidad abierta, resulta difícil exigir el cumplimiento de una responsabilidad definida. En otros términos, la responsabilidad en estos casos deja de ser una obligación de responder concretamente ante al guien por lo hecho, para tornarse un acto ético supererogatorio demasiado fuerte para ser requerido por una ética convencional. Por estas razones, parece plausible substituir el principio de responsabilidad por otro: el principio de protección que guía los actos de un modo más comprometido al mismo tiempo que viable.

\section{La ética de protección}

La ética de protección tiene sus raíces filosóficas en el propio origen del vocablo ética, cuyo sentido más arcaico es precisamente de "guarida" para animales y, por extensión, de "refugio y protección" para los seres humanos (Chantraine, 1968; Liddell \& Scott, 1968).

El principio de protección está en el fundamento del Estado mínimo que reconoce su obligación de cautelar la integridad física y patrimonial de sus ciudadanos a partir del siglo XVIII, siendo por ende también el fundamento moral del Estado do bienestar contemporáneo. Como es sabido, Ia salud pública nace en el siglo XVIII con el propósito de dar protección sanitaria a la fuerza laboral. Siendo así, el principio de protección subyace, desde hace por lo menos tres siglos, a las acciones públicas, tanto políticas como sanitarias, por lo que es sorprendente que la bioética no haya explícitamente incorporado este principio en su agenda.

Entendemos por protección la actitud de dar resguardo o cobertura de necesi dades esenciales, es decir, aquellas que deben ser satisfechas para que el afectado pueda atender a otras necesidades $u$ otros intereses.

Para poder hablar de un principio de protección y distinguirlo de otros principios, conviene asignarle por lo menos las siguientes características:

- Gratuidad, en el sentido de no existir un compromiso a priori de asumir actitudes protectoras;

- Vinculación, en el sentido de que una vez libremente asumida se convierte en un compromiso irrenunciable;

- Cobertura de las necesidades entendidas desdeel afectado.

De lo anterior se desprende que el principio de protección no es reducible al de beneficen- 
cia o a algún tipo de paternalismo. La legitimidad moral de la beneficencia depende de la evaluación del afectado que es el único que puede decidir si un acto será para él beneficente. En el caso del paternalismo es el agente quien decide lo que es beneficioso para el afectado, en independencia o aún en contra de la opinión de dicho afectado. Por lo tanto, estos principios difieren substancialmente de lo que aquí entendemos por protección.

Tareas de la salud pública son las medidas colectivas de prevención y promoción de la salud referentes a poblaciones humanas y a sus entornos socio-ambientales por un lado, y a instituciones públicas responsables por la implementación de políticas sanitarias legítimas y eficaces, por otro. El Estado debe asumir obligaciones sanitarias que implican una ética de la responsabilidad social correspondiente, que Ilamamos aquí de ética de protección.

La protección sanitaria puede ser vista como una propuesta de cuidar a la ciudadanía, con miras a prevenir enfermedades y fomentar un medio ambiente saludable. La protección no se confunde con el paternalismo beneficente pues, en principio, el agente protector no puede actuar sin el consentimiento de la población, debiendo proponer medidas públicas necesarias y razonables para prevenir los problemas sanitarios. Como ya señalado, el principio de responsabilidad no puede ser aplicado de forma operativa a acciones colectivas ni a políticas sanitarias por cuanto tanto los agentes como los afectados no pueden ser identificados. El principio de protección, en cambio, ofrece la posibilidad de una evaluación ética de acciones destinadas a cubrir necesidades sanitarias impostergables, efectivamente sentidas por la población.

La introducción del principio de protección para la evaluación moral de políticas públicas en salud colectiva será por ende bajo las siguientes condiciones concretas:

- Corresponde considerar la protección cada vez que determinados objetivos sanitarios son públicamente aceptados como mandatorios por estimarse indispensables.

- La aceptación de programas de salud pública implica la certeza - o la al ta probabilidad de que las medidas propuestas sean necesarias y suficientemente razonables para prevenir los problemas sanitarios abordados.

- Una vez aceptado como pertinente, el principio de protección puede cumplir su rol cabalmente, ya no pudiendo ser desestimado por razones secundarias, en vista que existe una necesidad social de ejercer la protección a través de las acciones programadas; vale decir, los eventuales efectos negativos no invalidan el programa.

Las políticas de protección de salud pública aparecen como propuestas frente a necesidades sanitarias colectivas. Una vez aceptadas, se vuelven obligatorias y permiten que el cumplimiento de las medidas de resguardo valide ejercer un legítimo poder de disciplina y autoridad para que sean efectivas. También se justifica someter la autonomía individual a los requerimientos del bienestar colectivo, en principio sin contemplar excepciones. La legitimidad de las acciones sanitarias y la inevitable restricción de la autonomía individual constituyen características de los actos protectores; la protección adquiere así una forma de poder sui generis, avalado por el principio de responsabilidad y caracterizado, como señalado, por la postergación de obligaciones frente a los individuos. Es decir, la justicia sanitaria prevalece sobre la autonomía individual.

Validada y aceptada la eficacia de un programa sanitario, es razonable suponer que el principio de protección, a diferencia del tradicional principio de responsabilidad, no implica en cada caso concreto exigencias y legitimaciones adicionales acerca de resultados esperados, riesgos, complicaciones o falencias, ya que todo ha sido previsto y explicado en el momento de la planificación, presentación y aceptación del programa. En caso de no ser aceptable, el programa ha de ser revisado y renegociado, siempre bajo la premisa que los requerimientos individuales deben ceder ante las necesidades sanitarias del bien común.

\section{Conclusiones}

Lo específico de la reflexión en salud pública es que tanto agentes como destinatarios no son individuos sino instituciones y colectivos.

Las autoridades sanitarias deben asumir algún nivel razonable y efectivo de resguardo, de servicios para el cuidado de la salud, de prevención de epidemias y de otros importantes programas de salud colectiva, incluyendo la promoción. Así y todo, estas tareas significan someterse a alguna forma de cobro o exigencia por los resultados obtenidos.

Rescatando los contenidos morales del principio de responsabilidad, la protección es una versión actualizada de lo anterior pues recupera el sentido de responder voluntariamente a las necesidades de los otros y también se preocupa de la eficacia y de la efectividad de las medidas de protección sanitaria adoptadas. 
Como sugiere el presente trabajo, la evaluación puede mejor realizarse a partir de un principio de protección, el cual impone el deber de eficacia como condición necesaria para una potestad legítima de intervención, autorizada para exigir a la autonomía personal que se ajuste al bien común.

La ética de protección debe ser entendida como un compromiso práctico, sometido a alguna forma de exigencia social, con lo cual la protección se vuelve un principio moral irrevocable, puesto que agentes, afectados, tareas y consecuencias deben ser definidos.

No quedan de este modo resuel tas las dificultades éticas en salud pública, pero al menos se intenta esclarecer algunos conflictos teóricos y prácticos que se han dado, en particular, por la confusión conceptual entre el nivel privado y el público en el análisis de sus problemas morales. Esta confusión ocurre cuando, en forma acrítica, se extrapola el modelo principialista del ámbito interpersonal al colectivo.
Es muy probable que al gunos problemas y dilemas morales en salud pública, aparentemente insolubles en la configuración actual de las éticas aplicadas, lo sean no únicamente por su supuesta complejidad intrínseca sino ante todo por las inadecuadas herramientas conceptual es empleadas. Intentamos aquí mostrar las insuficiencias del modelo canónico del principialismo cuando se traslada del plano de las relaciones interpersonales al nivel de análisis institucional, así como la imprecisión del principio de responsabilidad cuando los agentes y los destinatarios no son claramente identificables. La protección completa y especifica la responsabilidad, y en ese sentido sugerimos introducir el principio de protección, que consideramos más apropiado para integrar responsabilidad moral y efectividad pragmática, respetando ya sea la pluralidad de necesidades y valores de las sociedades actuales como alguna forma justa y razonable de propiciar bienes sanitarios.

\section{Agradecimientos}

Este trabaj o fue realizado con el apoyo del Consel ho Nacional de Desenvolvimento Científi co eTecnológi co, entidad del Gobierno Brasileño que apoya el desarrollo científico y tecnológico.

\section{Referencias}

BEAUCHAMP, T. L. \& CHILDRESS, J. F., 1994. Principles of Bi omedical Ethics. 4th Ed. Oxford: Oxford University Press.

BECKER, M. H., 1986. The tiranny of Health Promotion. Public Health Review, 14:15-25.

CALLAHAN, D., 1998. False Hopes. Why America's Quest for Perfect Health Is a Recipe for Failure. New York: Simon \& Schuster.

CHANTRAINE, Y. P., 1968. Dictionnaire Éthymologique de la Langue Grecque. Paris: Kliencksieck.

CLOUSER, K. D. \& GERT, B., 1990. A critique of principlism. Journal of Medicine and Philosophy, 15: 219-236.

COYE, M.J., 1994. Leadership in Public Health. New York: Millbank Memorial Foundation.

DANIELS, N., 1985. Just Health Care. Cambridge: Cambridge University Press. 
EVANS, J.-H., 2000. A sociological account of the growth of principlism. Hastings Center Report, 30: 31-38.

FERRATER-MORA, J., 1999. Dicci onario de Filosofía. Barcelona: Ariel.

GILLON, R., 1996. Principles of Health Care Ethics. Chichester: John Wiley.

GOFFI, J.-Y., 1997. Jonas Hans. In: Dictionnaire d'Éthique et dePhi losophie Morale (M. Canto-Sperber, ed.), pp. 763-765, Paris: Presses Universitaires de France.

JONAS, H., 1979. Das Prinzip Verantwortung. Versuch Einer Ethik Für DieTechnologische Zivilisation. Frankfurt am Main: Insel Verlag.

JONSEN, A. R. \& TOULMIN, S., 1988. TheAbuseof Casuistry. A History of Moral Reasoning. Los Angeles: University of California Press.

KUCZEWSKI, M., 1998. Casuistry and principlism: The convergence of methods in biomedical ethics. Theoreti cal Medicineand Bioethics, 19:509-524.
LACHM ANN, P. J., 1998. Public health and bioethics. Journal of Medicine and Philosophy, 23:297-302.

LEVINAS, E., 1974. Autrement qu'Être ou Au-delà de I'Essence. The Hague: Nijhoff.

LIDDELL, H. G. \& SCOTT, R., 1968. A Greek-English Lexicon. Oxford: Clarendon Press.

SKRABANEK, P., 1990. Why is preventive medicine exempted from ethical constraints. Journal of Medical Ethics, 16:187-190.

SKRABANEK, P., 1992. The poverty of epidemiology. Perspectives in Biology and Medicine, 35:182-185.

SKRABANEK, P., 1994. The Death of Humane Medicine and the Rise of Coercitive Healthism. London: The Social Affairs Unit.

THE NATIONAL COMMISSION FOR THE PROTECTION OF HUMAN SUBJECTS OF BIOMEDICAL AND BEHAVIORAL RESEARCH, 1978. The Belmont Report: Ethical Principles and Guidelines for the Protection of Human Subjects of Research. Washington, DC: U. S. Government Printing Office. 